\title{
Zur Kenntniss der menschlichen Placenta.
}

Von

\author{
Dr. F. N. Winkler, \\ pract. Arzt in Dresden.
}

(Mit Abbildungen Tafel V.)

In einer früheren ${ }^{\text {I }}$ Arbeit hatte ich den Bau der Placenta nicht eingehender schildern können, weil sich mir alle früher üblichen Härtungsmethoden als ungenügend erwiesen hatten. Bald war das Gewebe zu brüchig, bald zu weich geworden, bald wieder so verändert, dass nachträgliche Tinction nicht mehr statthaft war, kurzum ich kam bei den zunächst für mich selbst bestimmten Studien zu gar keinem Resultate.

Inzwischen versuchte ich eine andere von $R$ indfleisch sehr warm empfohlene Präparationsweise, nämlich die Tränkung der Gewebe mit Leim oder Gummi arab. und nachträgliche Härtung in Alkohol. Diese Methode hat sich mir auch für die Placenta durchaus bewährt, und ich kann sie mit grossem Nachdruck empfehlen.

Des Näheren war das Verfahren folgendes: Ich schickte zunächst eine Injection der Fötalgefässe mit durch Berliner Blau gefärbter möglichst concentrirter. Leimlösung voraus. Dass die

1) Textur, Structur und Zellleben in den Adnexen des menschl. Eies. Jena 1870. 


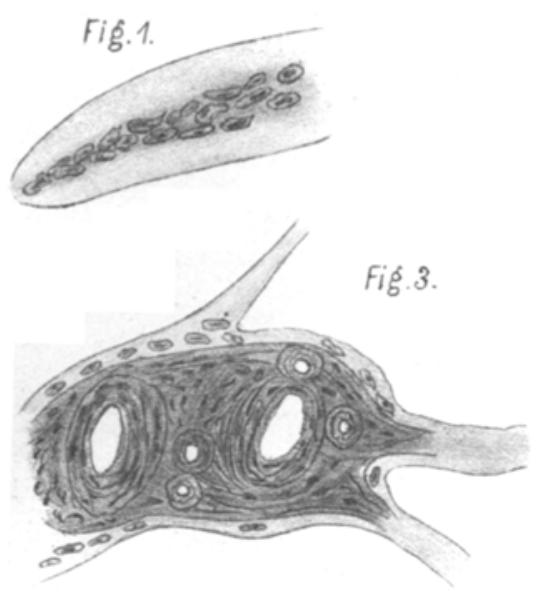

Fiह̂. 5.

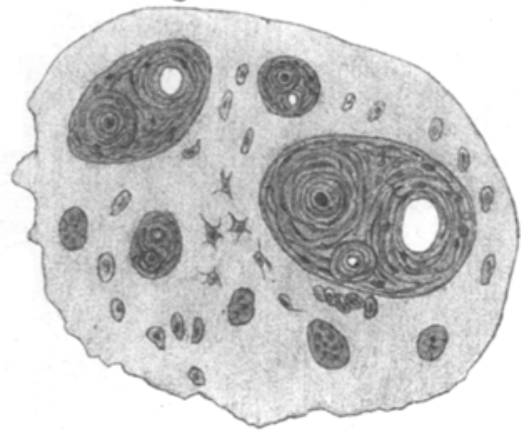

Fig. 7.

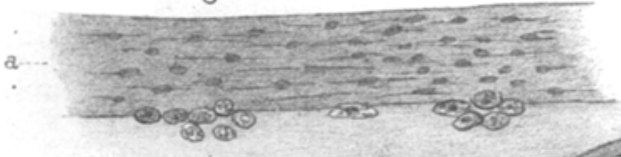

b...

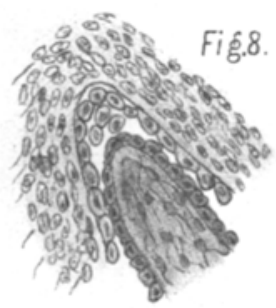

Winklen, dei,

Arohivf. Gynäkologie Bd.N.
Fig.2.
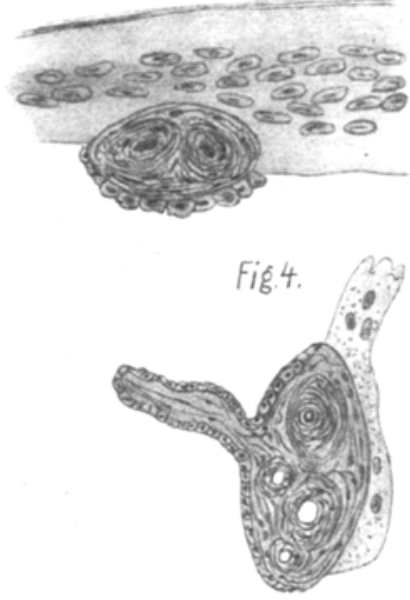

Fig.6.

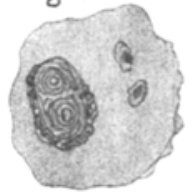

Schema des

Placentarbaves

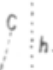

s.

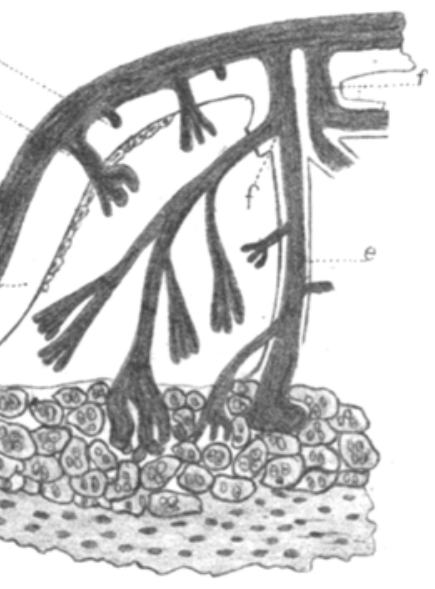


Leimlösung heiss, die Spritze gewärmt und die Placenta in warmem Wasser während der Injection suspendirt sein muss, versteht sich von selbst. Die Injection geschah von der Nabelvene aus : um das Blut aus den Fötalgefässen möglichst hinauszutreiben, wurden, weil der Druck der Nabelstrangarterien, die ja gewöhnlich bis zum völligen Verlust ihres Lumens contrahirt sind, ein viel zu hoher ist, die Arterien am Fusse der Nabelschnur angeschnitten, damit hier während der Injection das Blut ausfliessen konnte. War die Injection so weit gelangt, dass aus diesen Oeffnungen Injectionsmasse ausfloss, so wurde vermittelst Umstechung je eine Ligatur angelegt. - Unter häufiger Erneuerung des warmen Wassers, in dem die Placenta suspendirt war, wurde die Injection sehr langsam ausgeführt (Daver $1 / 2-3 / 4$ St.), die Zahl der etwa 50 C.C. haltenden Spritzen betrug bis zu acht. Dass sich die Zottenenden zu füllen beginnen, merkt man an dem die mütterlichen Bluträume in Folge der Volumenzunahme der Zotten verlassenden Blute. Von diesem Zeitpunkte ab darf die Injection nur sehr langsam vor sich gehen. Tritt aber dennoch Ruptur auf der Uterinfläche der Placenta ein, so war letztere entweder zur Injection überhaupt nicht geeignet, oder es trifft nur die Ungeduld des Experimentators die Schuld. - Nach beendeter Injection wurde unterbunden und die Placenta vorsichtig in Alkohol $(96 \%)$ gelegt, welcher letztere, weil er durch Wasserentziehung aus der Placenta sich sehr verdünnt, in den. ersten $24 \mathrm{St}$. etwa 4-6 Mal, in den nächsten drei bis vier Tagen aber etwa noch zweimal zu wechseln ist. Dann werden aus der Placenta Würfel mit etwa 1,5 Cm. $\square$ Fläche ausgeschnitten und nach kurzer Auswässerung in Eiswasser in die Gummi-Glycerinlösung für etwa 24 St. eingelegt, endlich wieder für 1-2 Tage in Alkohol geworfen, und sie sind nunmehr zum Schneiden prakticabel. Zur Herstellung der Gummilösung verrieb ich Gummi arab. mit möglichst wenig warmen Wassers zuerst und setzte dann Glycerin semper terendo hinzu, bis die Lösung dick fadenziehend wurde. Die Lösung muss aber ganz klar sein, zu welchem Behufe man sie am besten etwa 24 Stunden abstehen lässt. Von so präparirten Placentarwürfeln lassen sich prächtige Schnittchen machen, wie sie mir bei keiner anderen Methode frïher gelangen. Die Schnittchen wurden zunächst gut ausgewässert und dann auf 2-3 St. in ganz dünne Carminlösung gelegt. Nach dieser Zeit sind alle Fötalgewebe ziemlich stark, mütterliche Gewebe dagegen nur schwach und fast nur in ihrem zelligen Antheil tingirt. Beiderlei Gewebe 
treten somit in einen ziemlich schroffen Gegensatz der Farbennüancen zu einander. - Die Schnittchen müssen horizontal und auch vertical geführt werden und zwar sowohl besondere jedesmal für Basal- als auch für superficielle Schichten der Placenta. Das Resultat ist folgendes.

\section{Mütterlicher Antheil.}

In dem Gemisch von fötalem und mütterlichem Gewebe, wie es als Mutterkuchen geboren wird, ist der den Bau dieses Gebildes unmittelbar bestimmende Antheil das mütterliche Gewebe. Nach Abzug aller Fötalgebilde lässt sich der Rest immer noch als der durch seinen $\dot{B}$ au charakteristische Kuchen denken; nicht so aber umgekehrt. Die Fötalgebilde, für sich gedacht, sind Nichts als ein Convolut mehr oder weniger verzweigter Zotten, ohne jeden architektonischen Einfluss auf den Bau der. Placenta, in welche sie einfach nur so eingelassen sind, dass ihre Verzweigung an den durch das mütterliche Gewebe gegebenen architektonischen Grundriss und Durchschnitt durchaus gebunden erscheint. Für die Anschauung bezüglich der Architektonik der fertigen Placenta ist der gesammte fötale Antheil so vollständig bedeutungslos, dass ich, um letzteren geradezu mit dem Charakter eines architektonischen Ballastes zu belegen, die Ausdriicke Bruttoplacenta und Nettoplacenta benutzen will. Bruttoplacenta bezeichnet somit den factischen, wie er geboren wird, Nettoplacenta den ideellen Mutterkuchen, wie er nach Eliminirnng des gesammten fötalen Antheils erscheinen würde.

Die Nettoplacenta hat einen cavernösen Bau: Hohlräume, die mit einander communiciren und deren Scheidewände im Querschnitt das placentare Balkengerüst darstellen. Uterinwärts findet dies Gavernensystem (Pars cavernosa) seinen Abschluss durch die gewöhnlich als Serotina (im engeren Sinne) bezeichnete Basalplatte, fötalwärts aber durch die Schlussplatte. Zwischen beiden. Platten ist im Querschnitt das placentare Balkengerïst als ein, allerdings enorm weitmaschiges, Netz befindlich, in welchem zahlreiche verticale und, von diesen wieder ausstrahlend und den Zwischenraum zwischen beiden Platten in Stockwerke theilend, horizontale und schräge Balken verlaufen. Die Communicationsöfinungen zwischen den einzelnen Cavernen scheinen überaus gross zu sein, denn man erhält in Querschnitten zumal von den hori- 
zontalen Balken so gewöhnlich nur kurze Stücke, dass höchstwahrscheinlich die schiefe Schnittfiuhrung nicht allein beschuldigt werden darf.

Es sind somit zu unterscheiden:

A) Basalplatte (parauterin) (im Schema: c und d).

B) Schlussplatte (subchoriotisch) (im Schema: b).

C) Pars cavernosa (intermediär) (im Schema: e).

A) Die Basalplatte, von Dohrn, Langhans u. A. kurzweg mütterliche Placenta genannt - eine Benennung, die zu fortwährenden Verwirrungen Veranlassung giebt - besteht aus zwei Schichten, a) uterinwärts einer kleinzelligen, b) fötalwärts einer grosszelligen Schicht (s. im Schema: d und c). Die kleinzellige Schicht enthält einen vorwiegenden Antheil von Intercellularsubstanz und relativ spärliche eingesprengte ganz kleine Zellen. Die Intercellularsubstanz ist meist homogen, nur hier und da streifig, und zwar parallel der Basis, macht im Ganzen einen frischen Eindruck und nimmt lebhafte Carmintinction an. Freilich sind stets intensiver gefärbt die Zellen, an denen niemals auch nur die geringste Verfettung wahrgenommen wird. Die Dicke dieser Schicht taxirte ich auf ca. 0,1-0,2 Cm. - Innerhalb dieser Schicht findet stets die Ablösung der reifen Placenta Statt, wenn auch mit der Einschränkung, dass in den centralen Partien der Placenta diese Schicht in voller Dicke an der Placenta haften bleibt, selbst bis zur vollständigen Bloslegung der Uterusmuskulatur, in den äusseren peripherischen Theilen der Placentarbasis dagegen gewöhnlich die Ablösung keine vollständige ist, so dass entweder nur Theile oder selbst die gesammte Kleinzellenschicht am Uterus haften bleibt, so dass dann die Grosszellenschicht der Basalplatte der Placenta frei liegt. Die Ablösung der Placenta innerhalb dieser Kleinzellenschicht, oder in der Haftlinie dieser Schicht am Uterus, beweist, dass der Zusammenhang dieser Gewebstheile ein weit lockerer ist, als z. B. in der Grosszellenschicht, und der Umstand, dass nicht bloss reife, sondern auch unreife Placenten sich innerhalb dieser, wie Hegar betonte, während der ganzen zweiten Hälfte der Gestation stets vorfindenden Schicht ablösen, muss auf die Lehre von der Lockerung der Placenta berichtigend einwirken. Lockerung der Placenta war früher ein Dogma, und ist es heute leider immer noch für Manche. Die Erklärung war früher sehr einfach, denn man freute sich der regelmässig eintretenden Verfettung in der Grosszellenschicht, bis der Nachweis, dass für die 
Ablösung diese Grosszellenschicht ïberhaupt gar nicht in Frage kommt, sondern einzig und allein oder wenigstens vorwiegend die Kleinzellenschicht, in welcher zu jeder Zeit der Schwangerschaft die zelligen Elemente völlig gleiche Beschaffenheit aufweisen, eine andere Erklärung für den Mechanismus der Placentarlösung erheischte. Histologisch ist somit durchaus kein Grund zur Annahme einer im Verlauf der Gravidität wirklich stattgehabten Loekerung vorhanden. Geschieht die Ablösung der reifen Placenta trotzdem leichter als die der unreifen, so müssen andere Verhältnisse die Ursache sein. Ich habe schon früher (l. c. S. 46) ein gewöhnlich ganz vernachlässigtes Moment hervorgehoben, nämlich eine in Folge zunehmender Bindegewebswucherung der Nettoplacenta sich steigernde Starrheit des Kuchens. Dass bei der die Ablösung der Placenta bedingenden Flächenverkürzung des Uterus eine schlappe Placenta sich leichter in Falten legen kann, als eine starre, letztere dagegen eher abspringen wird und somit eine in ihrer Basis stattgefundene Lockerung vortäuschen kann, das ist eine Anschauung, die auf so einfachen mechanischen Verhältnissen begründet ist, dass sie - wie mir schien - unmöglich auf Widerstand stossen durfte. Angesichts der Thatsache, dass reife Placenten leichter sich ablösen als unreife, müssen, um den Causalnexus herzustellen, a priori die zwei Möglichkeiten statuirt werden, 1) Lockerung oder 2) Zunahme der Starrheit. Die Lockerung als Vorgang darf nicht mit der Lockerheit als Zustand verwechselt werden: Die Lockerheit in der Kleinzellenschicht ist allerdings vorhanden, eine Lockerung aber im Verlaufe der Schwangerschaft müsste erst, was noch nie geschehen, bewiesen werden, ist aber dem oben Gesagten zufolge so unwahrscheinlich, weil durch kein histologisches Moment belegt, dass sie vorläufig nicht einmal mit dem Rechte einer überhaupt ventilirbaren Frage dasteht. Anders der Starrheitsgrad des Kuchens: dass der reife starrer sei, als der unreife, ist eine Thatsache, die wohl ohne Weiteres zugegeben werden muss: die Ursache davon könnte wohl discutirt werden und es könnte wohl in Frage gestellt werden, ob die von mir beschuldigte Zunahme des Bindegewebes in der Placenta die alleinige Ursache sei. Wie aber iiberhaupt das aus dem höheren Starrheitsgrade der reifen Placenta resultirende mechanische Moment als ein für die Lösung derselben völlig bedeutungsloses hingestellt werden kann, ist mir in der That unverständlich. Die Grosszellenschicht (b) enthält die bekannten, meist auch als Decidualzellen mitbezeichneten, grossen Elemente, in ein 
äusserst spärliches, im Querschnitt trabeculäres Gewebe eingebettet. Letzteres, häufig ebenso schwer wie Kittsubstanz in Epithellagern zu finden, kann doch meistens an den Rändern von zerzupften Gewebsfetzen, noch besser aber in recht feinen und carminrothen Schnittchen nachgewiesen werden. $\mathrm{Ob}$ dies Gewebe jede einzelne Zelle netzförmig oder nur Gruppen, Nester von Zellen höhlenartig umschliesst, konnte ich nicht unterscheiden. Mir schien mehr das letztere der Fall zu sein. Ueberaus spärlich nach der Kleinzellenschicht hin, ohne dass der Zusammenhang von beiderlei Geweben deshalb in Abrede gestellt werden soll, nimmt es fötalwärts an Mächtigkeit zu, und alsbald erscheinen die Bälkchen bestehend aus homogener Intercellularsubstanz mit eingesprengten kleinen Bindegewebszellen ron runder, spindel-, selten sternförmiger Gestalt. Dies sind wahrscheinlich dieselben Elemente, welche von den meisten Beobachtern der Neuzeit hier und da in der Grosszellenschicht neben den gewöhnlichen grossen Zellen gesehen worden sind. Mit Ausnahme der Cotyledonensepta, in welche auch noch auf kurze Strecken die grossen Zellen mit eindringen, besteht die gesammte übrige Nettoplacenta nur aus dem eben erwähnten Bindegewebe: Somit besteht das gesammte Balkengerüst sowohl, wie die Schlussplatte nur aus Intercellularsubstanz nebst eingestreuten Zellen und in toto erscheinen beide nur als Fortsetzung des bindegewebigen Antheils der Basalplatte, nicht nur weil sie die gleichen histologischen Elemente enthalten, sondern weil man in Verticalschnittchen oft genug die säulenförmigen Placentarbalken sich als unmittelbare Fortsetzung dieses Gewebes kann erheben sehen.

Das Schema für die Balkendurchschnitte des gesammten Placentargerüstes ist demnach ein höchst einfaches. Die eingelagerten Zellen erscheinen zwar an keine bestimmte Ordnüng gebunden, jedoch halten sie sich stets in einer gewissen Entfernung von den Cavernen selbst, so dass auf die, von mir durch Versilberung nachgewiesene Endotheltapete der placentaren Blutcavernen stets ein lichter, von Intercellularsubstanz gebildeter Grenzraum folgt und auf diesen erst die immer nur im Centrum der Balken belegenen Zellen. Dasselbe wiederholt sich in der relativ sehr dicken Schlussplatte, die ebenso ihren Gehalt an Zellen hat, dagegen den lichten Grenzraum nicht bloss nach den unter ihr liegenden Cavernen zu, sondern auch nach dem Chorion hin besitzt. Der Gehalt an Zellen ist ein sehr verschiedener: nur selten so reichlich, wie in Fig. 1 und 2, meist weit geringer, ja mitunter verschwindend klein, so 
dass die Balken, zumal die Schlussplatte, fast nur aus Intercellularsubstanz zu bestehen scheinen. Die Intercellularsubstanz erscheint zu allen Zeiten völlig homogen, sehr selten von schwachkörnigem Gefïge. Ebenso machen die Zellen selbst stets einen durchaus frischen Eindruck. Meist rund, oval, spindelförmig, selbst auch exquisit sternförmig, besitzen sie stets ein völlig klares Protoplasma nebst bläschenförmigem grossen Kern und Kernkörperchen. Nie gelingt es, weder an Zellen noch an Intercellularsubstanz jemals auch nur die geringsten Spuren degenerativer Vorgänge aufzufinden.

Die Schlussplatte schliesst als eine dicke Schicht die superficiellen Cavernen nach dem $\mathrm{Ei}$ zu ab und grenzt fötalwärts unmittelbar an die hier im Bereiche der Placenta ïberaus dicke Schicht des Chorion (die Deckplatte), oder vielmehr an dessen Epithelschicht: jenseits des Chorion folgt dann Gallertschicht und ganz zuletzt erst Amnion. Es dürfte aus Vorstehendem wohl klar sein, dass eine Beziehung des beschriebenen placentaren Balkengerüstes zum Amnion auch nicht als eine entfernte Möglichkeit sollte gedacht werden können. Trotzdem sagt Jassinsky'): „Durch secundäre Theilung der Cotyledonensepta entstehen kleinere, secundäre Cotyledonen, so dass das Gewebe des Muttertheiles sammat den von ihm entspringenden Scheidewänden tief in den fötalen Theil eindringt, ohne jedoch das Amnion zu erreichen." Wer in aller Welt kann sich eine diesen Worten congruente Vorstellung bilden?

Genau so wie das Balkengerüst nach unten in das bindegewebige Stroma der Grosszellenschicht übergeht, ebenso lässt sich der gleiche Zusammenhang über den Rand der Placenta hinaus nach der Decidua nachweisen. Die Grosszellenschicht der Placenta findet hier ihr vollständiges Analogon in den sogenannten Decidualzellen, die, wie ich früher schon hervorhob, gleichfalls in ein .analoges, wenn auch spärliches, bindegewebiges Stroma eingebettet sind,

Im placentaren Balkengerüst sind makroskopisch die Verticalbalken zunächst durch die Cotyledonensepta markirt: indessen, da bekanntlich die Cotyledonenbildung nur oberflächlich besteht, nach der Tiefe aber schon sehr bald aufhört, so findet man auch die Septa sehr bald in verschiedene Trabekeln auseinandergefasert, und

1) Virchow's Archiv Bd. 40. S. 342. 
neben den meist quer oder schräg abgehenden einzelne auch gerad fötalwärts ziehen. Die weitere Präparation ist bei der Zartheit der Bälkchen meist unmöglich, hat man aber zù derartigen Untersuchungen die Placenten nicht nur einzeln, sondern gleich dutzendweis verwerthet, dann laufen auch solche mit stellenweis recht derben Trabekeln unter, in denen man die Verticalbalken direct bis zur Schlussplatte verfolgen kann. So habe ich z. B, auch eine (injicirte) Placenta gefunden, in welcher das mütterliche Gewebe an zwei Stellen durchaus unvollständig in den Cavernenbildungsprocess hineingezogen war. Histologisch allen uibrigen Trabekeln gleichend, und nur wenig und nur sehr winzige Cavernen fuihrend, so dass hier und da noch geradęu mütterliche Blutgefässe als ectatische Capillaren erkennbar waren, sieht man dies Gewebe in einer Breite von 0,5-0,8 Mm. sich säulenförmig von der Basalplatte erheben und geradezu in die Schlussplatte übergehen. - Neben diesen durch die Cotyledonensepta repräsentirten, relativ derben Verticalbalken und unabhängig von ihnen giebt es noch eben solche, die mehr im Centrum der Cotyledonen von der Basalplatte aufsteigen, jedoch recht zart und spärlich $\mathrm{zu}$ sein scheinen.

Die placentaren Blutcavernen sind durchweg mit einem, überall da, wo nicht gerade grosse Zottenhaufen die Wandung durchbrechen und die* Deutlichkeit des Bildes beeinträchtigen, leicht sowohl im Querschnitt, wie in der Flächenansicht durch Versilberung nachzuweisenden Endothel ausgekleidet. Während an den internen Cavernen nur der Zufall ganz deutliche Bilder giebt - die ich jedoch, ausdrücklich sei es gesagt, wiederholt gehabt habe - wies ich schon früher nach, dass zum bequemen Demonstriren sich das Coronargefäss von frischen Placenten ganz besonders gut eignet, ebenso auch, was ich jetzt noch hinzufügen will, die Cavernenfläche der Schlussplatte, die oft genug völlig oder nahezu frei von Zotten gefunden wird; gleiches gelingt auch, wenngleich schwerer, an der Cavernenfläche der Basalplatte. Ich glaube, dass nach dieser erfolgreichen Anwendung der Versilberung die Streitfrage über etwaige zellige Auskleidung der mütterlichen Bluträume in bejahendem Sinne als erledigt anzusehen ist.

Von der Uterinseite gesehen, zeigt die Placenta in.ihrer Basalplatte, und zwar besonders in Kreuzungsstellen der Cotyledonensepta mehr oder minder grosse Oeffnungen, durch welche die Communication der placentaren Blutcavernen mit den Bluträumen 
des Uterus vermittelt wird. Von diesen Oeffnungen aus dringt man entweder sofort in die Placentarcavernen ein oder findet erst eine Art Gefässrohr bis zu 3 oder $4 \mathrm{Mm}$. Länge. Makroskopisch glaubt man hier eine wirkliche Getässwandung zu unterscheiden, die sich jedoch im mikroskopischen Querschnitt durchaus nicht bestätigt, sondern einfach nur als aus placentarem Gewebe bestehend ergiebt, wie es der Grosszellenschicht entspricht, nur mit dem Unterschiede, dass die Intercellularsubstanz bedeutend zunimmt. Nach dem Gefässlumen hin folgt wieder der lichte, aus Intercellularsubstanz bestehende Grenzraum, und endlich die Endotheltapete: also auch hier derselbe Bau, wie sonst an Placentarcavernen. Das gesammte histologische Detail erweist mit ziemlicher Sicherheit die Richtigkeit der Virchow'schen Ansicht, dass alle Gefässräume der Nettoplacenta aus ectatischen Capillaren mit consecutiver cavernöser Ausbildung bestehen.

In der Basalplatte wollen manche Autoren glatte Muskelzellen gesehen haben. Die Kleinzellenschicht enthält ganz bestimmt keine, da sie ganz gleichförmig die oben erwähnte- Zusammensetzung hat. In der Grosszellenschicht fand ich jedoch Bilder, die in der That an Muskelelemente.denken liessen. Um die grossen Gefässlumina gruppiren sich mitunter die Zellen unter bedeutender $\mathrm{Zu}$ nahme der Intercellularsubstanz geradezu schichtenweise, concentrisch das Gefässlumen umkreisend, doch in der Richtung der Septa geschichtet ausstrahlend. Diese Zellen erscheinen in Querschnitten, zumal wenn sie, was ihrer Dicke wegen oft vorkommt, selber mit angeschnitten sind, als langgezogene mehr oder minder schmale Spindeln, mit grossem, öfters deutlich spindelförmigem Kern. Behält man aber stets die Bilder im Auge, welche zerzupfte Muskelzellen der Uteruswand selber gewähren, nämlich relativ schmale, aber sehr lange Zellen mit schmalem, jedoch mehr oder minder langem, stets aber relativ kleinem Kern, so muss zunächst constatirt werden, dass durchaus keine Aehnlichkeit besteht. Ich will die Stäbchengestalt des Kernes nicht, wie Jassinsky, als entscheidendes Merkmal ansehen, viel wichtiger erscheint mir die relative Grösse der Kerne. Bei allen Elementen der Grosszellenschicht, sobald sie irgendwie auf glatte Muskelzellen verdächtig werden, ergiebt genauere Vergleichung zumal in carminrothen Schnittchen, dass der Kern stets relativ sehr gross ist, und 2/3-3/4 der gesammten Zelle einnimmt. Dieser Umstand war für mich bestimmend, alle Elemente, auch wenn sie in Folge der 
Schnittführung als langgezogene Spindeln erscheinen, nicht für glatte Muskelzellen, sondern für gewöhnliche, nur schichtweis geordnete Elemente der Grosszellenschicht zu erklären. Ob man: die Zellen mit Säure isoliren kann oder nicht, erscheint mir für die Beurtheilung der Natur der Zellen selbst völlig gleichgültig, da in erster Linie die Qualität des Zwischengewebes dafür massgebend sein wird. Das Zwischengewebe ist aber in beiden Fällen sowohl in der Grosszellenschicht, wie in der Uterinwand selbst keine blosse Intercellularsubstanz, sondern ein wirkliches seine besonderen Zellen führendes Substrat von bindegewebiger Natur. Freilich erscheint es in der Uteruswand vorwiegend gefasert, dagegen in der Grosszellenschicht hauptsächlich homogen, und nur selten eine Andeutung von Streifen zeigend. An Zerzupfungspräparaten tritt dieser Unterschied zumeist hervor. - Die Blutbewegung in der Placenta kann wegen der allseitigen Communication der Cavernen, weil hierdurch jede vorbestimmte Richtung aufgehoben wird, nur eine sehr unregelmässige sein. Bald vorwärts geschoben, bald stillstehend, bald auch wieder rückwärts fliessend, so müssen wir uns die Blutbewegung in einzelnen Theilen der Placenta denken, während benachbarte Partien gleichzeitig vielleicht gerade die entgegengesetzte Bewegung zeigen. Hier sind alle Bluträume nur capillar und es ist nur Willkür gewesen, das Kranzgefäss, welches die nämliche Bedeutung hat, Kranzvene zu nennen. Dieser Name muss fallen, zumal absolut keine Veranlassung zu der Annahme vorliegt, dass die Blutbewegung gerade in dem Kranzgefäss centripetal sei.

Die Grösse der einzelnen Cavernen scheint nicht unbeträchtlich zu schwanken, sinkt jedoch kaum unter ein gewisses Maass; so pflegen z. B. am Rande der Placenta, bei einer Verjüngung von etwa 1-0,75 Cm. Dicke keine Stockwerke mehr zu bestehen, so dass die dortigen Cavernen von Basal- bis zur Schlussplatte reichen. Andererseits hat es mir einigemal geschienen, als ob dieselben Grenzen selbst bei voller. Dicke der Placenta (etwa $3 \mathrm{Cm}$.) bestanden hätten, so dass hier ganz enorm grosse Cavernen vorlagen. Allerdings bestehen in letzterem Falle zumeist wohl 2 bis 3 Stockwerke von Cavernen übereinander.

\section{Fötaler Antheil.}

Auf der Schlussplatte liegt als Deckplatte ( $\mathrm{Hyrtl}$ ) das Chorion, als eine dicke Schicht von dem auch sonst dem häutigen Chorion 
zukommenden Gefüge, einerseits übergehend in die Nabelschnur und hier deren axiale Partie, d. h. Gefässe nebst der allernächsten Schicht Sulze bildend, andererseits sich fortsetzend in das allbekannte häutige Chorion.

In Querschnitten von Nabelschnüren etwa 9-10 Wochen alter Eier erkennt man deutlich, dass die später ganz gleichförmige Sulze zwei verschiedenen Quellen entstammt. Axial liegen die drei Gefässlumina eingehüllt in eine Gewebsmasse, die mit dem sie umgebenden zweischichtigen (Epithel und Bindegewebsstratum) Amnionmantel entweder noch gar nicht oder nur unvollständig zusammenhängt. Schon in diesem Stadium ist das Bindegewebe des Amnion in Nichts von dem aus der Allantois herstammenden zu unterscheiden und beide enthalten in eine völlig homogene Intercellularsubstanz Zellen eingebettet, von rundlicher, Spindelja selbst exquisiter Sterngestalt mit langen Ausläufern, die einerseits zahlreich bis an die freien Räume (Analogon der Gallertschicht) zwischen Allantoisaxe und Amnion heranreichen, andererseits im Amnion ebenso reichlich zum Epithel hinziehen. Das Amnion kann man an solchen Eiern von der Insertion der Nabelschnur her auf erkleckliche Strecken von dieser abstreifen, so dass der Antheil der Allantois als axialer Cylinder zurückbleibt.

Nach dem Rande der Placenta zu verdünnt sich die Deckplatte etwas, um dann am Rande selbst in das noch dünnere velamentöse Chorion überzugehen. Die Zotten sind am velamentösen Theil sehr spärlich und winzig, dagegen hart am Rande der Placenta, worauf schon Bidder jun. ${ }^{1}$ ) besonders aufmerksam machte, sehr dicht und dick. Alle diese Zotten aber sind völlig obliterirt, d. h. gefässlos. Im Querschnitt solcher Zotten sieht man die homogene, hellglänzende Intercellularsubstanz durchsetzt von den meist sternförmigen und dann auch exquisite Netze bildenden Zellen. Der Bindegewebsstock als solcher erscheint gewöhnlich nackt, ohne jede eigene Epithelschicht in das Decidualgewebe eingelassen, und nur vereinzelt finden sich Zotten mit eigenem, jedoch nur rudimentärem Epithelmantel.

Ziemlich ebenso zahlreich, wie am Rande der Placenta, gehen auch vom Placentartheil des Chorion Zotten ab; die sich sämmtlich in die Schlussplatte einsenken. Ich unterscheide drei Arten dieser Zotten:

1) Holst, Beiträge II. 
a) solche, die sich später nicht nur nicht fortentwickelt haben, sondern einfach obliterirt sind. Solche Zotten enden einfach in der Schlussplatte selbst, ohne dieselbe bis zu den Ca-. vernen hin zu durchbrechen. In jedem Verticalschnitt durch die Schlussplatte findet man mehrere solcher Zotten, stets aber ohne eigene Epithelhülle. Das Vorkommen solcher obliterirten Zotten ist nicht auf gewisse Orte beschränkt, sondern sie kommen fast iiberall in der Schlussplatte vor; sie finden sich auch in der allernächsten Nähe der gefässhaltigen und zu mächtigen Bäumen entwickelten grossen Zottenstämme.

b) Zotten, die zwar gefässhaltig sind, jedoch, da sie sofort in den superficiellen, unter der Schlussplatte gelegenen Cavernen enden, keine besonders dicken Stämme haben und schon nach etwa dreimaliger Theilung überall in die Zottenenden übergehen. Der Stamm dieser Zotten verläuft gewöhnlich fast horizontal auf mehr oder minder lange Strecken in der Schlussplatte, bevor die eigentliche Theilung beginnt. So lange diese Zotten innerhalb der Schlussplatte laufen, also von mütterlichem Gewebe umschlossen sind, sind sie stets frei von jeder Epitheldecke und gewissermassen nackt in das mütterliche Gewebe eingelassen. Erst die frei in die Cavernen hineinragenden Zottentheile, und nur diese allein, tragen den bekannten Epithelmantel.

c) Die dritte Art Zotten unterscheidet sich von der zweiten nur durch ihre mächtige' Entwickelung, da sie zur Füllung der tieferen Cavernen verwendet werden. Dem entsprechend finden sich durch Sprossenbildung an ihnen mindestens doppelt so viel Rangstufen der Aeste wie an der vorigen Art. Die Theilung ist bald gabelig, d. h. zwei- oder dreigabelig, bald geradezu büschelförmig, letzteres zumeist für die Zottenenden. Die Rangstufen werden durchaus nicht immer alle durchlaufen, so dass von dem dicken Stamm erster Ordnung sofort Stämmchen vorletzter Ordnung abgehen können. Diese Stämmchen gehen meist rechtwinklig $a b$, um sofort in benachbarte Cavernen einzudringen. - Ein ander Mal enden dicke Stämme höherer Ordnung, indem sie der Auflösung bis zu Zottenenden entgehen. Zumeist findet dies Statt bei Zottenstämmen dritter, vierter Ordnung, die unter fortwährender seitlicher Abgabe von ganz kleinen Stämmchen bis an den Boden der Basalcavernen vorgedrungen sind. Hier dringen sie auch noch etwas in die Basalplatte ein und enden einfach abgerundet, oder kolbig verdickt, als ob sie hier keine zwingenden Bedingungen mehr für ihre Fortentwickelung gefunden hätten. 
Dies sind die von Langhans jünst beschriebenen dicken Zottenstammenden. Manche dieser Stämmchen verlaufen noch in der Basalplatte auf kurze Strecken, und dann unter Abgabe von unten in die Basalcavernen eindringender Zottenäste, oder aber sie lösen sich auch in Endzotten auf ohne die Basalplatte (und zwar deren Grosszellenschicht) zu verlassen. - Ein dritter Modus der Endigung ist noch der, dass ein Stamm vorletzter oder drittletzter Ordnung, gleichsam auf einer Vorstufe der büschelförmigen Theilung stehen gebliebẹn, keine wirklichen Fortsätze mehr absendet, sondern nur als eine 'mit mehr oder minder vorspringenden Buckeln besetzte Keule imponirt. -

Auch diese Zotten c) sind alle, soweit sie überhaupt in mütterlichem Gewebe liegen, völlig nackt: Spuren eines Epithelmantels, und zwar nur ganz unvollständig, sah ich in weit über hundert einzelnen Präparaten nur ein einziges Mal. Findet man, dass ein Zottenstamm einen weiten und langen Raum einer grösseren Caverne $\mathrm{zu}$ füllen hat, dann sieht man die Zertheilung der letzten drei oder vier Rangstufen der Zottenstämme innerhalb der Caverne selbst vor sich gehen und diese Theile der Stämme sind es, welche man in Zerzupfungspräparaten mit schönem Epitelmantel erblickt (oder auch ohne ihn, was dann Maceration oder die Zerzupfung selbst geleistet hat); denn alle Zottenpartien, die frei in die Caver-. nen hineinragen, besitzen ihre vollkommene Epitheldecke. -

Schneidet man an der Stelle, wo man die grossen Choriongefässe sich in die Tiefe senken sieht, eine Placenta ein, so erblickt man zunächst einen dicken, ganz hellgelben Stamm, der sich nach kurzem Verlauf alsbald büschelartig in etwa 8-12 relativ sehr dünne Aeste theilt. Langhans beschrieb dieses Verhältniss unter Beigabe einer klaren schematischen Zeichnung ${ }^{1}$ ) sehr gut, nannte jedoch diese Stämme (in dem Schema Lit. b) kurzweg Zottenstämme; mit wie wenig Recht, werden wir sofort sehen. Horizontale Querschnitte dieser Stämme (auf oben erwähnte Weise hergestellt und tingirt) lehren sofort, dass der Gehalt an mütterlichem Gewebe ein ganz vorwiegender ist, ferner dass die Zottenstämme in verschiedener Zahl und sehr verschịedener Dicke stets unter Verlust ihres Epithels in dieselben eingelassen, aber fest mit dem mütterlichen Gewebe verbunden sind. Neben diesen wirklich noch fungirenden Zottenstämmen finden sich in den obersten Theilen

1) Dieses Archiv Bd. I. Taf. V. Fig. I. 
dieser Placentarbalken noch in grösserer Zahl winzige, völlig obliterirte Zöttchen. Macht man Schnitte aus immer tieferen Theilen, selbst schon unterhalb der büschelförmigen Theilung, so sind die Bilder im Grossen und Ganzen stets dieselben: entweder die Zottenstämmchen liegen frei in Cavernen und besitzen ihren Epithelmantel, oder sie liegen eingebettet in mütterliches Gewebe, in Placentarbalken und sind nackt.

Die Einlagerung dieser Zottenstämme in die Placentarbalken ist meist axial, doch auch excentrisch, und selbst peripherisch. In letzterem Falle trägt dann die der Caverne zugewandte Fläche des Zottenstammes ihr normales Epithel, während andererseits die Verbindung mit dem mütterlichen Gewebe stets die gewöhnliche und feste ist, so dass selbst intensiver Druck auf das Deckgläschen nicht leicht eine Dehiscenz herbeiführt. Dieselbe feste Verbindung der entblössten Zottenstämme mit dem mütterlichen Gewebe, obwohl stets unter Festhaltung besonders in Carminpräparaten sehr scharf markirter Grenzen, besteht allïberall. Die Fläche, in welcher beiderlei Gewebe fest mit einander verbunden sind, ist somit sicher eine sehr bedeutende und es sind nicht bloss die winzigen Haftstellen der Langhans'schen Zottenstammenden an der Basalplatte, welche die Verbindung vermitteln.

Obschon es aus manchen Analogien keine fremde Erscheinung ist, dass an einer unter Druck seitens eines anderen Gewebes stehenden Membran deren Epithelschicht usurirt wird, so muss man dennoch, da gewöhnlich geglaubt wird, dem Eie sei durch eine zusammenhängende Epithelschicht gegenuiber dem mütterlichen Organismus eine gewisse Selbständigkeit garantirt, überrascht werden durch das umfangreiche Fehlen dieser schützenden Epitheldecke. Ich möchte hier gewissermassen die Achillesferse des ganzen Eies annehmen, die Heerstrasse für den Import vieler krankmachender Einflüsse und hereditärer Momente, letzteres etwa in dem Sinne, dass die Einwanderung specifisch imprägnirter Zellen eine bedeutende Erleichterung findet.

Die Zottenstämme sind in ihrem Verlaufe durchaus an die Placentarbalken gebunden, und zwar ist in den meisten Balken fötales Gewebe vorhanden. Es ist immerhin als ein nicht häufiger Befund zu verzeichnen, wenn man Placentarbalken auf mehr oder minder grosse Strecken frei von jeglichem Gehalt an Zotten findet.

Das placentare Chorion (Deckplatte) haftet der Schlussplatte 
gewöhnlich ziemlich fest an, obschon die Verbindung keine so innige zu sein scheint, wie die der Zottenstämme selbst. In Querschnitten tritt weit leichter auf Druck Dehiscenz ein, ausserdem finden sich, wenn gleich nie in zusammenhängender Lage, hier und da in der Grenzlinie Zellen vor, die stets in weit vorgeschrittener fettiger Degeneration sich befinden. Ich halte diese Zellen für Rudera des früheren Chorionepithels, erstens weil ich nicht weiss, was dieselben zum mütterlichen Gewebe überhaupt für eine Beziehung haben sollten, und dann, weil sie dem sonstigen Chorionepithel in der, häufig noch gut erkennbaren Würfelgestalt der einzelnen Zellen durchaus gleichen. Von einem verticalen Einschnitt durch die ganze Dicke der Placenta aus kann man mit dem Scalpelstiel das Chorion von der Schlussplatte häufig in ziemlichem Umfange abtrennen, stets unter Abreissung kleinerer Zöttchen, dagegen überzeugt man sich sofort an den Stellen, wo sich grössere Zottenstämme in Verticalbalken der Placenta einsenken, von der überaus festen Verbindung dieser Theile.

Das placentare Chorion besitzt durchaus keine eigenen Capillaren. Sämmtliche Gefässe passiren einfach das Chorion, um nach der Schlussplatte and durch diese hindurch mindestens in die superficiellen Cavernen als Zottengefässe vorzudringen. Trotzdem aber muss hervorgehoben werden, dass ganz gewiss einzelne der kleinen Gefässe schon innerhalb des Chorions, wenn auch nur auf kürzere Strecken, durchaus den Charakter von Capillaren tragen, an denen gewöhnlich noch eine Spur von Adventitia nachzuweisen ist. Man kann sich hiervon in guten Injectionspräparaten nach Abtrennung des Chorions von der Schlussplatte überzeugen. Capillarnetze aber sind, wenigstens an der reifen Placenta, sicher nicht vorhariden. Ueber die Angaben von Jungbluth, der constant Capillarnetze im placentaren Chorion bis zur Mitte der Gestation fand, fehlt es mir fast an allen Erfahrungen.

Die Richtigkeit dieser Angaben vorausgesetzt, glaube ich wohl, dass die Beziehung dieser Capillarnetze zum Fruchtwasser eine innige, ebenso die der Persistenz jener Netze zum Hydramnios eine nahe sei. Die Capillarnetze, um die Mitte der Gestation verschwindend, finden ihren Ersatz in den Saftcanälchen, die nach meinen Erfahrungen etwa um dieselbe Zeit ihre Persistenz erlangen. Die Saftkanälchen durchziehen die Chorionbindegewebsschicht, die Gallertschicht und auch das Amnion, dessen Epithel unter Läckenbildung durchbrechend, so dass sie nach der Eihöhle 
frei ausmünden, genau so wie Saftkanälchen auf der Serosa des Diaphragma. Die Saftkanälchen im Chorion lassen sich zwar bis an dessen Epithelschicht heran verfolgen, jedoch erscheint es für den velamentösen Theil wegen der unmittelbar darauf folgenden degenerirten Deciduaschichten unwahrscheinlich, dass die Saftkanälchen von hier direct ihre Füllung beziehen sollten: es ist wohl eher anzunehmen, dass die vegetative Richtung in den Saftkanälchen hierselbst mehr nach dem Chorionepithel hin, als von ihm her vor sich geht. Die vorwiegende Bezugsquelle glaube ich in dem placentaren Theil des Chorions, sowie in der Nabelschnursulze suchen zu müssen. Die Verbindung der Saftkanälchen mit Blutgefässbahnen findet man allerwärts, mit Gefässen von sehr verschiedenem Caliber, und zwar sowohl mit Arterien, als auch mit Venen; wenn auch vorwiegend mit ersteren. An feinsten Capillaren ist die Verbindung als eine Durchbrechung der Capillarwand Seitens der Saftkanälchen nicht schwer zu erkennen: diese Ueberzeugung gewinnt man am leichtesten an lebenswarmem Material, d. h. im Heiztisch mit feuchter Kammer. ') Experimentell ist diese Thatsache für andere Gewebe schon durch Carter erledigt, der von Capillaren aus Injectionsmasse in die Saftkanälchen getrieben hat. An Gefässen höheren Ranges scheint die Verbindung keine so unmittelbare zu sein, wenigstens gelingt der Nachweis nicht. Die Saftkanälchen gehen aussen bis an das Gefässrohr deutlich heran: ob sie hier noch tiefer gehen, oder einfach münden, so dass eine Art von Lymphraum um das Gefäss herum entsteht, muss ich ganz unentschieden lassen. Solche Verbindungen der Saftkanälchen kommen selbst mit den sehr weit calibrirten Nabelstranggefässen durchgehends vor.

Reitz' ${ }^{2}$ lässt die Zottengefässe in perivasculären Räumen flottiren. Dem muss ich einfach widersprechen. Diese Räume sollen am mächtigsten an den Zottenenden sein, also voraussichtlich hier am leichtesten zu erkennen. Nun liegen die Capillaren aber hier gewöhnlich in solchen Knäueln, dass die Schlingen sich gegenseitig überdecken und eher Alles andere, nur kein klares Bild gewähren. Mit diesen Zottenenden ist Nichts zu beweisen.

1) Zur Aufklärung von Missverständnissen bezüglich meiner Arbeit: „Textur, Structur" u. s. w., sei ergänzend erwähnt, dass der Ausdruck ,lebenswarm" stets die Untersuchung im Heitztisch involvirte. Der von mir benutzte Tisch stammt von F. Heinitz in Wien.

2) Stricker, Handbuch d. Lehre v. Geweben. S. 1185. 
Hat man frische Zotten, ohne jede künstliche Injection, im Querschnitt, so findet man nie Anhaltspunkte für die Annahme perivasculärer Räume. Ist aber erst durch Maceration auch das Endothel der Capillaren abgelöst, dann können allerdings hierher bezügliche Bilder entstehen. Auch schon die Thatsache, dass man öfters ein Saftkanälchen bis in das Endothel der Capillaren vordringen sieht, widerspricht geradezu der Statthaftigkeit einer Annahme von pericapillären Räumen. Anders liegt diese Sache für die grösseren Gefässe. Für diese erscheint mir die Annahme perivasculärer Räume nicht unwahrscheinlich, erstens weil die Saftkanälchen sich stets nur bis an das Gefässrohr heran verfolgen lassen und dann weil Injectionen in das Parenchym ganz besonders gern längs der Gefässe fortkriechen und drittens weil im placentaren Chorion nicht selten, hart den Gefässen anliegend, Cysten vorkommen, die, von etwa bis Bohnengrösse, äusserst dünnwandig und von einer grünlichen colloiden Flüssigkeit gefüllt sind. Sie sind ausgekleidet von einer schönen Endotheltapete und sind, wie ich schon in einer früheren Arbeit erwähnte, einfach als cystös degenerirte perivasculäre Lymphräume aufzufassen.

Auf alle.Fälle stellen die Saftkanälchen Bahnen dar, in welchen wenigstens Flüssigkeit leicht vorbewegt werden kann. Wenn aber Jungbluth die Persistenz des Capillarnetzes im placentaren Chorion als Ursache von Hydramnios beschuldigt, so klingt diese Hypothese a priori sehr wahrscheinlich. Dabei muss als selbstverständlich angesehen werden, dass in solchen Fällen auch gèwissermassen ein Hydrops der Gallertschicht gefunden werden müsse. In einem exquisiten. Fall von Hydramnios complicirt mit fötaler Rachitis ${ }^{1}$ ) fand ich 'aber im Gegentheil eine sehr spärliche Gallertschicht, ein ganz normales Chorion, auch im placentaren Theil keine Abweichung, dagegen im Amnion eine sehr bedeutende Ectasie der Saftkanälchen, eine Abnormität, die sich bis in die Nabelschnursulze hinein verfolgen liess. Dieser Fall ist zwar kein Gegenboweis gegen die Jungbluth'sche Ansicht, ergiebt jedoch immerhin soviel, dass eine Persistenz der Chorioncapillaren durchaus nicht die einzige Ursache von Hydramnios sei, und dass in diesem Falle, gleichviel ob die Saftströmung in den ectatischen Saftkanälchen centrifugale oder centripetale Richtung hatte, von sämmtlichen Eihäuten nur das Amnion in nähere Beziehung zum $\mathrm{Hy}$ dramnios gebracht werden durfte.

1) Dieses Archiy II. S. 101. 
Was die sonstige Gefässvertheilung im Chorion anbelangt, so kann ich eigentlich nur das Bekannte bestätigen. Für diese Untersuchung sind nicht nur vollkommen injicirte, sondern namentlich auch unvollkommen gefüllte Placenten nothwendig, in denen Venen und Capillaren jedoch nur bis zu den Arterien hin gefiullt sind. Die circuläre Muskelschicht an Arterien ist stets von auffallender Mächtigkeit und bedingt es, dass nach dem durch die ersten Inspirien so colossal herabgesetzten Aortendruck die sämmtlichen arteriellen Gefässe sich bis zum völligen Verlust ihres Lumens, und zwar tonisch für die Dauer - schliessen; der frühere Inhalt der Arterien, durch die Capillaren hindurch in die Venen getrieben, und, weil auch letztere durch eine, wenn auch schwächere Contraction der relativ weit geringeren Muskellagen sich verengen, dem Kinde zugetrieben wird, zumal der durch die Inspirien bedingte negative Druck seine, öfters bis auf $8 \mathrm{Cm}$. vom Nabel aus noch sicht- und fühlbare Saugkraft äussert. Dieses Quantum Blut kommt somit dem Kinde zu Gute, besonders da letzteres wegen der plötzlichen Füllung des weiten Lungenkreislaufs sich zeitweilig gleichsam in einem Zustande wie nach einer rapiden arteriellen Blutung befindet. Dieses Stadium der artériellen Anämie kann erst eintreten, nachdem überhaupt schon aus anderen Gründen Inspirien stattgefunden haben. Ich glaube Symptome eines dieser arteriellen Anämie entsprechenden Dyspnoe nicht selten gefunden zu haben: nach den ersten relativ ruhigen Inspirien sieht man öfters plötzlich, namentlich bei kräftigen Kindern, deren erste Inspirien gleich energisch ausfallen, plötzlich die Inspirien den Charakter der höchsten Dyspnoe annehmen. Die ausgiebigsten nur denkbaren Reflexe kommen zur Anschauung: Krampfhafte Contractionen der Inspirationsmuskeln an Brust und Hals, weites Aufreissen des Mundes, Herabzerren und Umwälzen der Unterlippe, Reflexe der Arme wie zum Festhalten, der Beine wie zum Feststemmen, der Thorax wird enorm stark elevirt. Diese stürmischen Erscheinungen pflegen eine Zeit lang anzuhalten, bis das Stadium der arteriellen Anaemie durch Ausgleichung auf dem Wege der Circulation beendet ist, und es folgen nunmehr ruhige, regelmässige Inspirien mit meist rein abdominellem Typus. Bisweilen werden Kinder blau-asphyctisch geboren, machen gleich einige Inspirien und verfallen dann ganz plötzlich, gleichwie ohnmächtig geworden, in das Stadium der Asphyxia pallida. Auch hier glaube ich die arterielle Anämie beschuldigen zu müssen, welche wegen der schon bestehenden Decomposition der Blutgase um so intensiver 
auf das Gehirn und Respirationscentrum einwirken muss. In diesen Fällen ist das Schwenken nach Schultze vorzüglich, nicht nur weil es den Luftwechsel in den Lungen sowie die Circulation vermittelt, sondern auch weil es bei Verlängerung der elevirten Haltung (Kopf abwärts, Exspirationsstellung) das Blut zum Gehirn gravitiren lässt.

Angesichts dieser Erwägungen muss es als dringend nothwendig erscheinen, die Unterbindung der Nabelschnur nicht nur nicht gleich nach der Ausstossung der Frucht, sondern sogar erst nach Ausstossung der Placenta vorzunehmen, weil inzwischen die durch sofortiges Massiren gesteigerten Uteruscontractionen durch Vermehrung des intracapillären Druckes in den Zotten voraussichtlich noch die Ueberführung eines gewissen Quautums Blut zum Kinde herbeigefuihrt haben werden. Will man nicht erst auf die Ausstossung der Placenta warten, so muss jedenfalls als Zeitpunkt für die Abnabelung nicht das blosse fühlbare Aufhören der Pulsation, sondern vielmehr das Erblassen des Stranges und zumal die völlige Entleerung der Nabelvene abgewartet werden. Jedes Plus an gesammter Blutmenge kann nur als Gewinn für das Neugeborene, als eine Erhöhung seiner Widerstandsfähigkeit gegen die auf dasselbe einstürmenden consumirenden Einflüsse angesehen werden, und ich möchte fast glauben, dass die Abuabelung in der vorgeschlagenen Weise, consequent durchgeführt, sehr wohl auch in einem Fallen der Ziffer für die bekannte Gewichtsabnahme Neugeborener zur Geltung kommen dürfte. Diese Erwägungen erschienen mir um so wichtiger, weil gerade in Anstalten, wo Wägungen allein gemacht werden, in dem Geschäft des Abnabelns gar zu leicht eine gewisse Ueberstürzung einreisst, eine Erfahrung, die man fast allerwärts machen kann. -

Ueberall innerhalb der Zotten, selbst in deren Stämmen finden sich zahlreiche allseitige Anastamosen, daneben in den Zottenstämmen selbst zahlreiche langmaschige Capillarnetze, die theils um die Gefässstämme gelagert, theils einfach in das Parenchym eingesenkt erscheinen. Daher findet man im Querschnitt eines Zottenstammes ausser den Gefässstämmen fast immer noch mehrere kleinere Gefässlumina. Ausserdem findet man, als ziemlich constante Erscheinung, dass ein 'ganzer Büschel von Endzotten stets nur von einem einzigen, langgezogenen Capillar versehen wird, welches einfach durch locale Verlängerung je eine Schlinge für jede Zotte bildet und offenbar in derselben Weise in jede etwa 
neu sprossende Zotte Schlingen hineinschickt. Die innerhalb dieser Schlingen in einer Zotte vielleicht vorhandenen anastamotischen Queräste denke ich mir, wie ich Aehnliches schon früher für die Allantoiscapillaren des Schaafes beobachtete, durch den Durchbruch einer Sternzelle gleichzeitig nach beiden Armen der ursprünglichen einfachen Schlinge entstanden. Aus der Kenntniss dieser vielfachen Anastamosen geht hervor, dass die Circulation zeitweilig über ganze Strecken gestört oder gar gehemmt sein mag, und damit hängt auch die Erfahrung zusammen, dass selbst bei den allerbesten Injectionen gewöhnlich einzelne Theile der Gefässbahn, zumal im Capillargebiete von der Injectionsmasse völlig freibleiben. Findet man in Präparaten gerade die Grenze der injicirten Capillaren, so erscheint deren Fortsetzung, sobald sie, weil ohne Inhalt, zusammengefallen sind, ganz so wie Saftkanälchen, sehr gering an Breite, jedoch an vielen Stellen mit einem dunkeln axialen Streif, als optische Wirkung der Lichtung des Rohrs, und dann auch häufig mit doppelt conturirter Wandung versehen. Oft schon frappirten mich im ersten Augenblicke derartige Bilder dermassen, dass ich hier einen directen Zusammenhang mit Saftkanälchen erkennen zu müssen glaubte, bis die Kanälchen sich nachträglich als ungefüllte Capillaren declarirten. Ausserdem fand ich auch bei schwacher Füllung der Capillaren mit Injectionsmasse Bilder, die auffallend an injicirte Saftkanälchen erinnerten und schliesslich doch nur Capillaren waren. Unter solchen Umständen würde ich einer etwa behaupteten Injection der Saftkanälchen in Zotten von deren Capillaren aus keine vollgültige Beweiskraft beilegen können, da der Einwurf, wenig oder gar nicht injicirte Capillaren für Saftkanälchen gehalten zu haben, nicht vollständig entkräftet werden kann.

Bezüglich der Entwickelung bin ich wegen Mangel an ausreichendem Material völlig ausser Stande, eine ergiebige Darstellung zu liefern. Der einzige Punkt, den ich denke sicher erkannt zu haben, ist das Hineinwachsen der Zotten in die Utriculardrüsen.

Ich behandelte die Placenta eines normalen, in Folge eines Trauma ausgestossenen, 9 wöchentlichen Abortiveies nach der oben erwähnten Methode und fand in hart unter dem Chorion geführten Flächenschnitten, dass der mütterliche Antheil aus einem kleinzelligen Gewebe bestand, in welchem viele freie Lumina enthalten 
waren, die alle ihre eigene epitheliale Auskleidung besassen. Dieses Epithel ist einschichtig und besteht aus grossen, vollsaftigen Zellen, die öfters sogar so schmal und lang sind, dass sie von Cylindergestalt erscheinen. Die Lumina selbst sind rund oder bei schiefem Schnitt länglich im Querschnitt. In diesem Lumen liegen Zottenstämmchen, von dem eigenthümlichen, einschichtigen Zottenepithel in durchaus vollständiger Lage bedeckt und in Nichts von den sonstigen Eigenschaften der Zotten unterschieden. Indem aber diese Zottenstämmchen die Lumina nicht vollständig ausfüllen, gewähren sie in dem freien Zwischenraum die Möglichkeit zu einer mehr oder weniger dichten Ansammlung von Zellen, die allem Aussehen nach deutlich Drüsenelemente sind und den Epithelzellen jener Räume, in welchen die Zotten lagern, durchaus gleichen. - Ausser diesen Zottenstämmchen giebt es noch andere, winzige Zotten, die gleichfalls ihren normalen Epithelmantel tragend, einfach in mütterliches Gewebe eingelassen sind, ohne aber in mit eigenem Epithel ausgekleideten Hohlräumen zu lagern. Entweder sind dies Zotten, die selbständig, ohne Drüsenlumina zu berühren, in mütterliches Gewebe eingewachsen sind, oder sie sind Aeste von Zottenstämmen, die nach Durchbrechung der Drüsenwandung direct in mütterliches Gewebe eindringen. Dass letzteres vorkommt, findet man oft genug in Schnittchen, erstere Möglichkeit aber muss als ganz unwahrscheinlich hingestellt werden, weil diese Art von Zöttchen je weiter uterinwärts, desto reichlicher werden. Wären 'diese Zöttchen sēlbständig in mütterliches Gewebe eingewachsen, so müsste ihr reichlichster Fundort die Schlussplatte selbst sein, nicht aber umgekehrt. Es muss demnach angenommen werden, dass die Zottenstämme in Uterusdrüsen hineinwachsen, und dann unter Durchbrechung der Drüsenwandung ihre Aeste direct in mütterliches Parenchym hineintreiben. Hier wachsen sie so lange fort, bis sie auf Bluträume stossen, in welche sie derart eindringen, dass sie die einzelnen Zellen des die Bluträume auskleidenden Endothels auseinanderdrängen und durch die so in der Endotheltapete entstandenen Lücken direct in die Blutcavernen eindringen. Da an reifen Placenten die Zottenstämme überall nackt im mütterlichen Gewebe liegen, so müssen in Folge des gegenseitigen Druckes zweierlei Epithelien zum Schwund gelangt sein, einmal das Zottenepithel selbst und dann das Epithel der Utriculardrüsen. Bis zu diesem Zeitpunkt, an welchem auch eine feste Verbindung der nackten Zottenstämme mit mütterlichem $\mathrm{Ge}$ webe zu Stande kommt, liegen die Zottenstämme ganz lose in 
Kanälen der Decidua und lassen sich wie der Klingeldraht in der Wand hin- und herbewegen. So sah ich einmal an einem 12 wöchentlichen Ei (das ich, aus dem letzten Kriege zurückgekehrt, leider vertrocknet und verdorben vorfand) die Decidua enorm hypertrophirt und durch sie hindurch Zottenstämme von etwa $3 \mathrm{Cm}$. Länge ganz in der eben beschriebenen Weise laufen.

Das ist so ziemlich Alles, was ich mit Sicherheit über das Verhalten der Utriculardrüsen fand. Uebrigens gehen aus den früher mitgetheilten Thatsachen noch folgende Schlüsse hervor. Bezüglich der Regeneration der Drüsen an der Placentarstelle muss ich im Gegensatz zu manchen Autoren, z. B. Friedländer, annehmen, dass von Drüsen an der Uterusinnenfläche absolut Nichts haften bleibt. An Stellen, die den centralen Partien der Placenta entsprachen, fand ich die Uterusinnenfläche entblösst bis auf die Muskulatur, deren innerste Schichten noch cavernöse und ectatische Capillaren enthielten. In der Placenta selbst fand ich in keiner Schicht der Basalplatte solche Spuren der Utriculardrüsen, wie Jassinsky; ebensowenig lassen sich solche in der Pars cavernosa entdecken. Bei den Hunderten von Präparaten, die ich gemacht, fand ich, sobald das Material ganz frisch und ohne jede natürliche oder sogenannte methodische Maceration zur Untersuchung kam, alle Zottentheile, die frei in Cavernen flottirten, mit der einfachen, gewöhnlichen Zelllage bedeckt. Doppelte Epithellagen, trotzdem die Zotten stets nicht nur möglichst, sondern absolut ohne jede Läsion untersucht wurden, habe ich niemals gesehen und fürchte, dass ebenso, wie ich früher die homogene Schicht auf dem Zottenepithel als Kunstprodukt bedingt durch die Maceration in Salzsäure (Jassinsky) erklärt habe, auch die doppelte Zelllage, wie sie Jassinsky abbildet, gleichfalls nur ein auf irgend eine Weise zu Stande gekommenes Kunstprodukt sei. Sollte etwa die äussere Zellschicht dem wirklichen optischen Querschnitt entsprechen, und die innere Zelllage aus einer anderen optischen Ebene der walzenförmigen Zotte stammen? Freilich sträubt man sich gegen eine derartige Verdächtigung des Autors; jedoch erscheint auch die andere noch übrig bleibende Möglichkeit sehr unwahrscheinlich. Da nämlich nach Jassinsky die Endzotten grösserer Zottenbäume mit diesem doppelten Epithel bedeckt sein sollen, so müsste eine enorme dendritische Wucherung der Drüsen angenommen werden, was meiner Erfahrung widerspricht, da die Zotten aus den Drüsen unter einfacher Durchbrechung ihrer 
Wandung anstreten. Als regelmässiger Befund sind somit die Jassinsky'schen Angaben ganz sicher nicht zutreffend, aber selbst als seltene Ausnahmen nach dem eben Gesagten sehr unwahrschëinlich.

\section{Verkalkungen.}

$\mathrm{Zu}$ allererst hebe ich hervor, dass Verkalkung an der normalen, reifen Placenta nicht wie man früher annahm, nur in $5-10 \%$ (nach Carus) vorkommt, sondern ein nahezu ebenso constanter Befund ist, wie die fettige Degeneration. Unter 43 Fällen fand sie sich nur $2 \mathrm{Mal}$ nicht. Damit soll aber nicht gesagt werden, dass sie in diesen 2 Füllen wirklich nicht existirte. Denn die Verkalkungen sind mitunter schwer zu finden. Gewöhnlich erkennt man sie bei guter Beleuchtung der Uterinfläche schon mit blossem Auge und fühlt bei leichter Messerführung während, des Einschneidens ein deutliches Knirschen. Bei geringerer Verkalkung stechen die verkalkten Partien immer noch von der wegen ihrer Verfettung allerdings gleichfalls weisslichen Serotina einigermassen $a b$ : bei den geringsten Graden aber fehlt auch dies Criterium. und ich habe in einzelnen Fällen auf gut Glück 10-20 Schnittchen aus der Serotina gemacht, ehe ich winzige Kalkdeposita fand. In einem Falle fand ich auf 20 Schnittchen nur eine einzige Concretion und ich halte es nur für Zufall, dass ich bei den oben erwähnten 2 Placenten keinerlei Concretionen fand. Je grösser die Ueberzengung vom constanten Vorkommen der Kalkdeposita, mit desto mehr Sorgfalt wird man ihnen im einzelnen Falle nachspüren und sie desto sicherer auch überall finden. Der gewöhnliche Fundort der Concretionen ist die Basalplatte und zwar deren Grosszellenschicht. Demnach liegen dieselben an der geborenen Placenta nicht frei 'zu Tage, sondern sind noch von der Kleinzellenschicht als einem dünnen, ziemlich durchsichtigen Häntchen bedeckt. Der in mikroskopischer Beurtheilung der Placenten Geübte weiss in Einschnitten meist schon mit blossem Auge zu erkennen, dass zumeist nur mütterliche Gewebstheile verkalkt sind: natürlich muss dann die mikroskopische Untersuchung nachfolgen, bei der sich geringere Betheiligung fötalen Antheils überhaupt erst ergiebt. In der Basalplatte erscheinen die Concretionen plattenförmig, von hier aus ziehen etwa verkalkte Zottenstämme (Langhans'sche) als Kalkwalzen in die Pars cavernosa placentae hinein. Häufig verkalken nur solche Partien des fötalen Antheils, 
die innerhalb der Grosszellenschicht selbst belegen sind. Wenn aber behauptet wird, dass die Verkalkungen niemals die ganze Dicke der Placenta durchsetzen, so muss ich dem widersprechen. In einem Falle, den ich in der Sammlung des Gebärhauses zu Jena deponirt habe, liefen enorm zahlreiche Kalkbalken durch die ganze Placenta bis zur Schlussplatte hin, und zwar verliefen diese Balken horizontal, schräg und vertical, die letzteren auch öfters bis in die Basalplatte vordringend. In diesem Falle waren Mutter, Kind normal. Die einzige Abnormität, die sich vielleicht dadurch erklären lässt, dass von sämmtlichen Zottengebilden nach der Schätzung mindestens der zehnte Theil verkalkt war, bestand darin, dass die Apnoe des in Steisslage befindlichen. Kindes auffallend rasch und, wie es während der Geburt schien, ganz unmotivirt beeinträchtigt wurde. -

Unterm Mikroskop setzt man zur Entkalkung Säuren hinzu und beobachtet genau den Vorgang der Entkalkung. In vielen Fällen reicht Ac: acet. aus, in anderen muss mit Acid. mur. nachgeholfen werden, in noch anderen Fällen löst Ac. acet. fast gar Nichts (nur sehr spärliche Gasblasen tauchen auf) und nur Ac. mur. bedingt die Lösung. Somit erscheint kohlensaurer Kalk immer, öfters sogar. als einziger Bestandtheil, Phosphate"dagegen kommen nie ganz selbständig vor, obwohl öfters in weitaus vorwiegender Menge. Die Bestimmung der, verkalkten Gewebe ist oft recht schwer: denn nach vollendeter Entkalkung unterscheiden sich diese Partien in Nichts von den nicht verkalkt gewesenen. Den eigenthümlichen Glanz, wie ihn Langhans hervorhebt, habe ich nicht finden können: jedenfalls aber ist diese Eigenschaft nicht so prägnant und constant, dass man darauf ein entscheidendes Criterium basiren könnte. Während der Entkalkung werden viele Präparate in Folge der colossalen Menge von Gasblasen durchaus undeutlich und unbrauchbar zur Beurtheilung der Frage, welche Gewebe ïberhaupt und welche vorwiegend verkalkt sind; die besten Bilder erhält man aus schwach verkalkten Partiẹn. Unter 23 darauf hin untersuchten Placenten war mütterliches Gewebe allein $8 \mathrm{Mal}$, mütterliches und fötales $12 \mathrm{Mal}$ und nur ein Mal bloss fötales Gewebe verkalkt. In 2 von diesen 23 Fällen -wurde kein e Verkalkung gefunden. Im mütterlichen Antheil beschränkt sich die Verkalkung in ihren Anfängen nur auf die grossen Zellen, und erst bei höheren Graden der Verkalkung scheint sich auch das intermediäre Bindegewebe zu betheiligen, denn die Ver- 
kalkungen erscheinen als grosse Kalkklumpen ohne jede unverkalkte Zwischensubstanz. Quetscht man solche Kalkklumpen, so zerbrechen sie stets in ganz unregelmässige Stïcke, was nicht möglich wäre, wenn das Substrat der grossen Zellen unverkalkt bliebe. In einzelnen Fällen pflanzt sich die Verkalkung auf das Balkengerüst der Pars cavernosa fort, und zwar hier bald mit, bald ohne Betheiligung der inliegenden Zottenstämme. Fötale Gewebe scheinen zumeist nur in der Nachbarschaft der Kalkherde des mütterlichen Gewebes zu verkalken. Auf diese Weise kommt es, dass einzelne der Langhans'schen dicken Zottenstammenden entweder nur in ihrem kolbigen Theile, oder auch noch etwas darüber hinaus verkalken. In diesen Fällen - und dies ist das häufigste - ist das mütterliche Gewebe bei Weitem am stärksten verkalkt: seltener findet sich die Verkalkung vorwiegend in Zotten und am seltensten in diesen allein. Die verkalkten Theile der Zotten sind gewöhnlich einzelne Zottenenden, oder auch Stämmchen niederster Rangstufen, soweit sie frei in Cavernen hineinhängen, seltener soweit sie in die Placentarbalken oder in die Grosszellenschicht eingebettet sind. Von den Endzotten sind immer nur einzelne verkalkt, die benachbarten und zu demselben Bäumchen gehörigen dann völlig normal. Die Verkalkung geht dann durchaus nicht auf den Stamm ïber. - In den Stämmchen selbst kann die Verkalkung entweder den ganzen Stamm oder nur einzelne Strecken desselben einnehmen, oder kann in durchaus unregelmässig zerstreuten kleinen Häufchen auftreten. Es ist mir nicht gelungen für diese Verkalkungen bestimmte Ursachen ausfindig zu machen, und ich muss annehmen, dass die Ursache überhaupt immer nur auf kleinere Zellengruppen ihren Einfluss beschränkt, somit durchaus keine allgemeine, sondern nur rein locale Bedeutung bat. Damit stimmen auch die verschiedenen Formen der Zottenverkalkungen, die bald centripetal, bald wieder centrifugal erscheinen. Man findet entweder bloss den Epithelmantel, oder ausser diesem noch mehr oder minder grosse Strecken des Bindegewebsstockes verkalkt, oder endlich nur die Capillarwandung, die alsdann von einem feinkörnigen Grau wie angehaucht, oder leicht rauchig erscheint. Meist sind trotzdem die Zottengetässe selbst noch völlig durchgängig und voll Blut, seltener erscheinen sie durch Kalkmassen verstopft, und dann greift auch die Verkalkung auf den Bindegewebsstock iiber.

Im Allgemeinen ist die Betheiligung der Zottengebilde an der Verkalkung eine so geringe, dass von einer Beeinträch- 
tigung der kindlichen Existenz nur äusserst selten die Rede sein kann.

Da ich oben gezeigt, dass sowohl mütterlicher Antheil allein, wie auch fötaler für sich verkalken kann, so scheint mir dadurch erwiesen, dass der Anstoss zur Verkalkung sowohl von der Mutter, als auch rom Kinde ausgehen kann: überwiegend ist aber der erstere. Ich hatte schon früher Zottenverkalkung als einen nur secundären Vorgang geschildert, der, wie auch Langhans schildert, meist dort, wo an der Basalplatte Zotten und Grosszellenschicht sich berühren, zu Stande kommt. Jedoch ist dies, wie oben erwähnt, nicht ausschliesslich der Fall: ebenso ist zu berichtigen, was Langhans sagt, dass er nie normale feine Zotten verkalkt gesehen habe und daher auch nicht glaube, dass die in die Basalplatte eingewachsenen feinen Zotten rerkalkten: so finde ich in meinen Notizen bei 3 Fällen ausdrücklich vermerkt, dass viele der eingewachsenen kleinen Endzotten verkalkt waren. Andererseits hingegen stimme ich mit ihm darin überein, dass von Fötalgebilden die letzten Enden der dickeren, in die Basalplatte sich einsenkenden Zottenstämme sehr häufig verkalken, wenn auch nicht gerade „vorzugsweise“. Langhans") schilderte ebenso, wie ich schon früher (1. c.) die Verkalkung des Epithelmantels der Zotten und zumal die centripetale Richtung der Verkalkung. Fränkel ${ }^{2}$ ) dagegen hob mit Nachdruck den Beginn der Verkalkung in der Zottencapillarwand hervor; die beigefügte Tafel ist recht naturgetreu. Seine Belegfälle sind 4; davon zwei Frühgeburten todtfauler Früchte, scheiden somit aus; sein Belegmaterial reducirt sich also auf 2 Placenten. Trotzdem lautet das Resumé höchst „entschieden“ über das „bei Weitem häufigere“ Vorkommen dieser oder jener Verkalkungsform. Jedoch hat Fränkel immerhin Recht, den Beginn der Verkalkung für viele Fälle in die Capillarwand zu verlegen - in 23 Fällen fand ich. 3 Mal exquisite Verkalkung von Capillaren kleinster Zotten, darunter 1 Mal bei 6 Wochen ante partum acquirirter Syphilis und inzwischen beendeter Schmierkur - und für solche Fälle die Quelle des Kalkes im fötalen Blute zu suchen, sicher aber nicht in der Weise, dass der Fötus sein Pensum der Knochenbildung mit der 36. Woche absolvirt habe und nunmehr seine ihm unbequemen Kalkschätze in die Zotten deportire. Zur Begründung dieser An-

1) Dieses Archiv I. S. 317.

2) Ebend, II, S. 373 . 
sicht stellt Fränkel zwei Behauptungen auf; erstens, die Knochenbildung des Fötus sei um diese Zeit beendet, was Niemand, der Knochenuntersuchungen gemacht hat, zugeben wird. Zweitens wird in Folge der ersteren Behauptung ein Kalküberfluss im fötalen Blute präsumirt, von dem aber noch keine chemischen Untersuchungen bislang Etwas wissen. Die Kalkdeposita finden sich nirgends im fötalen Gebiet als nur in Zotten, und Fränkel scheint sich dies dadurch zu erklären, dass die Placenta ohnehin in regressiver Metamorphose begriffen sei. Nun sind aber Placenta und Zotten durchaus zweierlei : zeigen sich constant an der Placenta regressive Vorgänge, so zeigen sich an Zotten fast ebenso constant keine und dadurch eben zeichnen sich sämmtliche Zottengebilde in der Placenta ganz besonders aus. Bedenkt man überhaupt die Unregelmässigkeit, mit der solche Kalkdeposita im fötalen Gebiet vorkommen, so kann man für gewöhnlich kaum andere, als rein lokale Ursachen annehmen, die auf einzelne Endzotten oder auf einzelne Zellengruppen der Zottenstämme beschränkt, hier die Kalkablagerungen vermitteln. Freilich mag das Auftreten dieser Ursachen wohl mit der Reifung des Eies in Zusammenhang stehen, aber nicht gerade weil im fötalen Blute Ueberschuss an Kalk vorhanden sei. Denn schon bei dem gewöhnlichen Kalkgehalte des Blutes wird beim Fötus mit derselben Leichtigkeit in dazu neigenden Zellgruppen Kalk sich ablagern können, wie c. p. im extrauterinen Leben, und es ist nicht einzusehen, warum gerade beim Fötuis ein Ueberschuss an Kalksalzen im Blute präsumirt werden müsse, eine Bedingung, die für extrauterines Leben ohne Weiteres gar nicht in Anspruch genommen wird.

Zum Schluss sei noch beiläufig erwähnt, dass unter den letzten 23 Placenten zwei bei Syphilis der Mutter und nach schon beendeter Schmierkur geboren wurden. Im ersten Falle, 8 Wochen a. part. acquirirt, keine Spur von Verkalkungen; im anderen 6 Wochen a. part. acquirirt, enorme Verkalkung. Der zweite Fall, in welchem keine Verkalkung gefunden wurde, betraf eine junge, völlig gesunde Person.

\section{Erklärung der Abbildungen.}

Schema des Placentarbaues.
a) Placentares Chorion (Deckplatte nach Hyrtl).
f) Zottenstämme nebst Verästelungen, als Fortsetzung von a). 
g) Kurze Zottenstämme, in oberflächlichen Cavernen endend.

h) obliterirte Zotten, gar nicht in Cavernen eindringend.

b) Schlussplatte rit Endothel.

e) Senkrechter Placentarbalken als Hülle um fötales Gewebe.

c) Grosszellenschicht Kleinzellenschicht Basalplatte.

Fig. 1-6. Placentarbalken:

Fig. 1. ohne Fötalgewebe.

Fig. 2. mit peripherischer Lage des Zottenstammes.

Fig. 3. mit dickem, centralem Zottenstamm.

Fig. 4. mit excent. Zottenstamm; mütterliches Gewebe fast verschwindend.

Fig. 5. mit dickem Stamm (im Schema bei $f$ ). Vergr. 20 .

Fig. 6. mit Zottenstamm und Resten seiner Epithelhülle.

Fig. 7. Chorion (a) nebst Schlussplatte (b); dazwischen Zellenreste. Stïck einer obliterirten Zotte (o).

Fig. 8. Zotte innerhalb eines Drüsenlumens. 9wöchentliches Ei. 